\title{
Innovación en docencia de sistemas de comunicación en el Grado de Ingeniería Telemática de la UVEG*
}

\author{
Jaume Segura $^{1}$, Carmen Botella ${ }^{1}$, Antonio Soriano ${ }^{1}$ y Santiago Felici ${ }^{1}$ \\ ${ }^{1}$ Dpt Informàtica - ETSE - Universitat de València.
}

\begin{abstract}
In teaching Communication Systems, we find a high theoretical component. The perception about the related subjects cause a negative perception in the students.

This work explains the motivation and the strategy followed to re-orientate this perception by introducing Software Defined Radio elements (like USRPs) in different subjects in the Degree and the Master in Telecommunication Engineering at the University of Valencia.
\end{abstract}

Keywords: USRP, RTL-SDR, communications, GIT, MITUV.

\section{Resumen}

En la docencia de sistemas de comunicación hay una componente teórica elevada. La percepción de los estudiantes al estudiar estas asignaturas es negativa.

Este trabajo explica la motivación y la estrategia seguida para reorientar esta percepción a partir de la introducción de elementos de Software Defined Radio y USRPs en diferentes asignaturas del Grado y del Máster de Ingeniería Telecomunicación de la Universitat de València.

Keywords: USRP, RTL-SDR, comunicaciones, GIT, MITUV.

\footnotetext{
*Proyecto financiado por la Universitat de València (UV-SFPIE_RMD15-314373)
} 


\section{Introducción}

La enseñanza en el Grado de Ingeniería Telemática (GIT) tiene una notable carga docente en sistemas de comunicación. Algunos autores (Blázquez I 1994) han tratado la integración curricular de las tecnologías de la comunicación en las aulas, estableciendo así una serie de criterios para esta integración, entre ellos encontramos: (1) el análisis la calidad de los recursos, que siendo de diferentes niveles permiten interaccionar con los sujetos, ya que de acuerdo con estos autores lo importante es esta interacción entre sujetos y recursos/medios; (2) la inserción de los contextos metodológicos adecuados, ya que un potente medio puede tener menos potencialidad si el método en el que se incluye no es acorde a los objetivos buscados; (3) la identificación de los destinatarios adecuados, ya que los recursos deben estar adaptados a las necesidades y capacidades de los estudiantes. De esta forma puede entenderse que a determinados niveles de maduración sea más viable el uso de recursos que otros; (4) la conducción del profesor, ya que los estudios empíricos demuestran que el conocimiento y la implicación de éste es uno de los factores decisivos para determinar la bondad del recurso.

Sin embargo, se debe tener en cuenta una serie de condiciones que comprenden que los recursos no sustituyen al profesor y que requieren un uso reflexivo, crítico y adaptado a la realidad de los estudiantes. Con todo ello, se debe contemplar la explotación de los recursos de innovación para que los estudiantes consigan un aprendizaje significativo (Aguaded-Gómez J I 2001).

De acuerdo con lo anteriormente expuesto, nuestro propósito en este artículo es explicar la motivación y el desarrollo metodológico basado en el uso de plataformas Software Defined Radio (SDR) para la docencia en el Grado de Ingeniería Telemática de la Universitat de València, así como analizar una prueba piloto que se está desarrollando en un proyecto de innovación educativa que ha sido financiado en parte por la Universitat de València. El resto del artículo se desglosa de forma resumida en las siguientes secciones: objetivo, metodología en la que se desarrolla el marco en el que se desarrolla el proyecto, desarrollos del proyecto que conllevan un estudio previo sobre la apreciación de los estudiantes, la descripción de la motivación y del material sobre el que se desarrolla el proyecto y la descripción de estas experiencias docentes.

\section{Objetivo}

El objetivo principal de este proyecto de innovación educativa es aumentar el grado de experimentalidad en las asignaturas de comunicaciones del Grado. Para ello necesitamos realizar una evaluación previa de la percepción de los estudiantes.

Como objetivos específicos se plantea el desarrollo y testeo de un conjunto de prácticas para consolidar conceptos relacionados con las comunicaciones digitales. 


\section{Metodología}

El desarrollo metodológico que ha motivado el proyecto que nos ocupa está basado en la apreciación que se tenía en los últimos años sobre el desarrollo teórico-práctico de los laboratorios de las asignaturas de Transmisión de Datos, Teoría de Comunicación, Comunicaciones Inalámbricas y Movilidad, del Grado de Ingeniería Telemática y de asignaturas que desarrollan contenidos más avanzados en el marco del Máster de Ingeniería en Telecomunicaciones. Los contenidos que se distribuyen en las diferentes asignaturas se desarrollan progresivamente especificando: diferentes modulaciones digitales (en banda base y pasabanda), diferentes técnicas de ecualización de canal para evitar interferencias intersimbólicas (ISI) monoportadora y multiportadora, técnicas de codificación de canal, técnicas de sincronización, técnicas de diversidad y de espectro ensanchado, etc.

Esta apreciación está refrendada por la encuesta que realizamos a estudiantes de estas asignaturas en el curso 2015-16, en que se les preguntó sobre la visión de las comunicaciones que ofrecían estas asignaturas en el marco del grado/máster y que trata de valorar la necesidad de una mayor carga experimental en la docencia sobre comunicaciones. Los resultados se analizarán más adelante.

Por otra parte, se plantea el desarrollo de esta carga experimental basada en Software Defined Radio (SDR). El concepto de SDR fue introducido por Joseph Mitola ( Mitola J 1992) y establece un nuevo paradigma educativo en el ámbito de las telecomunicaciones que permite implementar mediante software muchos componentes de sistemas de radiocomunicaciones y éstos pueden ser reconfigurados en línea. Con ello se consiguen plataformas hardware inalámbricas multi-estándar, multi-banda y multifuncionales. Este cambio de paradigma tendrá un impacto notable en la evolución de futuras comunicaciones inalámbricas y sistemas en red.(Mao Sh y col. 2013)

Algunas herramientas basadas en esta tecnología, como son las USRPs o RTL-SDR, son opciones abiertas, económicas y que ofrecen una versatilidad suficiente, que con un adecuado enfoque pedagógico pueden ser muy útiles para el estudio de estándares tecnológicos actualmente vigentes. Una Universal Software Radio Peripheral (USRP)(Ettus Research 2015) es una plataforma diseñada por Ettus Research (y actualmente vendida por National Instruments), basada en una arquitectura con FPGA y una capa de comunicaciones (daughter-board) que es intercambiable.

A este respecto, tomamos como referentes los casos de la Universidad de Cantabria, la Universidad de Sevilla (Pinar-Domínguez I y Murillo-Fuentes J J 2011) y la Universidad de Washington(Wyglinski A M, Pu D y Cullen D J 2011), que ya han incorporado estos elementos a su docencia.

La aproximación metodológica seguida en este proyecto se ha basado en el diseño de una serie de sesiones prácticas usando USRPs y GNU Radio Companion que desarrollan diversos conceptos de las asignaturas implicadas. Estas sesiones prácticas han sido experimentadas inicialmente por un grupo de estudiantes que han servido de población control durante todo el proyecto. 


\section{Desarrollos del proyecto}

Se aprecia que actualmente las asignaturas de comunicaciones del Grado de Ingeniería Telemática de la ETSE de la Universitat de València, que incluyen Fundamentos de Sistemas de Telecommunicación, Teoría de Comunicación, Transmisión de Datos y Comunicaciones Inalámbricas y Movilidad, tienen una carga experimental insuficiente. Por otro lado, los sistemas de telecomunicación a los que los estudiantes podrían acceder en base a los conocimientos que se plantean no son configurables o si lo son, tienen un coste fuera de las posibilidades presupuestarias de la escuela para los laboratorios. Por ello, el uso de sistemas diseñados mediante Software Radio se plantean como una solución viable para este propósito.

\subsection{Resultados del estudio previo a los estudiantes}

En base al análisis que se quiere realizar sobre la percepción que tienen los estudiantes sobre la docencia en las asignaturas de comunicaciones en el GIT, se les ha planteado una encuesta diseñada 'ad-hoc' para recoger las valoraciones que pretendemos analizar en el proyecto y que de acuerdo con algunas opiniones puntuales que hemos recogido anteriormente, esta apreciación global podría mejorarse mediante la introducción de cierto grado de experimentalidad en las asignaturas (no basado en resolución de problemas).

La encuesta está basada en un formulario que recoge las siguientes preguntas que són valoradas en una escala de 1 a 5 donde 1 es 1 es "Totalmente en desacuerdo" y 5 es "Totalmente de acuerdo" (la valoración 0 corresponde a NS/NC):

- Q1: ¿Las asignaturas de comunicaciones del GIT/MITUV ofrecen una visión de los sistemas de comunicaciones actuales?

- Q2: ¿Las asignaturas de comunicaciones del GIT/MITUV ofrecen unas prácticas adecuadas?

- Q3: ¿Valorarías positivamente la inclusión de cierta carga experimental en estas asignaturas que desarrollasen los conceptos que se explican en las asignaturas implicadas?

Finalmente, se pregunta sobre la apreciación de los estudiantes sobre si en estas asignaturas hay poca carga experimental. Si a esta pregunta se responde afirmativamente se pide en qué aspectos se cree que podría mejorarse y si se sabe qué es el SDR.

A partir de la encuesta hemos obtenido que el $50 \%$ de las respuestas las han dado chicos y el $50 \%$ chicas, siendo la totalidad de estudiantes de grado. Sobre la respuesta a Q1, hemos obtenido un valor promedio (y desviación estándar) de 3.2(1.2), por lo que los estudiantes consideran que las asignaturas implicadas ofrecen una visión suficiente de los sistemas de comunicaciones. Sobre Q2, su valor promedio 2.8(1.3), así los estudiantes tienen la visión que las prácticas no tienen suficiente carga experimental aunque en cierto grado puedan ser adecuadas y Q3 obtiene una valoración promedio 
de 4.2(1.0), por lo que (a criterio de los estudiantes) la introducción de cierta carga experimental ayudaría al desarrollo conceptual. Además, mayoritariamente consideran que las prácticas tienen poca carga experimental y abogan por la introducción de sistemas de comunicaciones reales.

\subsection{Planificación de las experiencias con USRPs y GNU Radio Companion}

Con el propósito de dar una base experimental al desarrollo conceptual de las asignaturas implicadas en el proyecto, se ha elegido la USRP como plataforma de desarrollo básica (aunque también pueden utilizarse RTL-SDR como plataforma receptora en la banda entre $30 \mathrm{MHz}$ y $1750 \mathrm{MHz}$ ).

\subsubsection{Descripción del material a utilizar}

El equipo (Ettus Research 2015) del que se dispone para el desarrollo de las prácticas estáá compuesto por:

- 5 plataformas USRP N210

- 1 plataforma USRP2

- 2 plataformas USRP1

- 2 daughter-boards LFTX y 2 LFRX (entre 0 y $30 \mathrm{MHz}$ )

- 1 daughter-boards BasicTX para USRP N210 (entre 1-250MHz)

- 2 daughter-boards XCVR2450 para USRP1 (con banda dual entre 2.4-2.5GHz y 4.9-5.9GHz)

- 2 daughter-boards CBX-40 para USRP N210 (entre 1.2-6 GHZ, con hasta 40 $\mathrm{MHz}$ de ancho de banda)

- 2 antenas verticales VERT2450 (entre 2.4-2.5 y 4.9-5.9 GHz) y 2 antenas LP0965 Log-Periódicas PCB (entre $850 \mathrm{MHz}$ y $6.5 \mathrm{GHz}$ )

\subsubsection{Desarrollo de experiencias docentes y propuestas de laboratorios}

Para desarrollar conceptualmente el currículum que implican las asignaturas anteriores, se ha elegido el siguiente conjunto de prácticas orientadas a este desarrollo conceptual:

- Lab1: Introducción al uso de GNU Radio Companion. Simulación de modulaciones 2D y multiportadora.

- Lab2: Introducción al uso de USRP con GNU Radio Companion. Realización de un sistema de emisión-recepción FM. 
Innovación en docencia de sistemas de comunicación en el Grado de Ingeniería Telemática de la UVEG

- Lab3: Estudio de modulaciones con USRPs.

- Lab4: Análisis de transmisión con OFDM

- Lab5: Estudio de estándares de comunicación (802.11a/g/p, DVB-T, RDS$\mathrm{TMC}, \mathrm{NFC}, \ldots)^{1}$

El Lab1 constituye una introducción conceptual a GNU Radio Companion, un software open-source, y que permitirá el aprendizaje de uso de este entorno como simulador. Con este entorno se ha preparado un estudio con simulación del error de bit con diferentes tipos de modulaciones, así como la simulación de un sistema OFDM. En la figura 1 vemos el esquema de un sistema de comunicación que modula con QAM y en la figura 2 vemos un esquema de simulación en el que se estudia las características de esta técnica de modulación multiportadora.

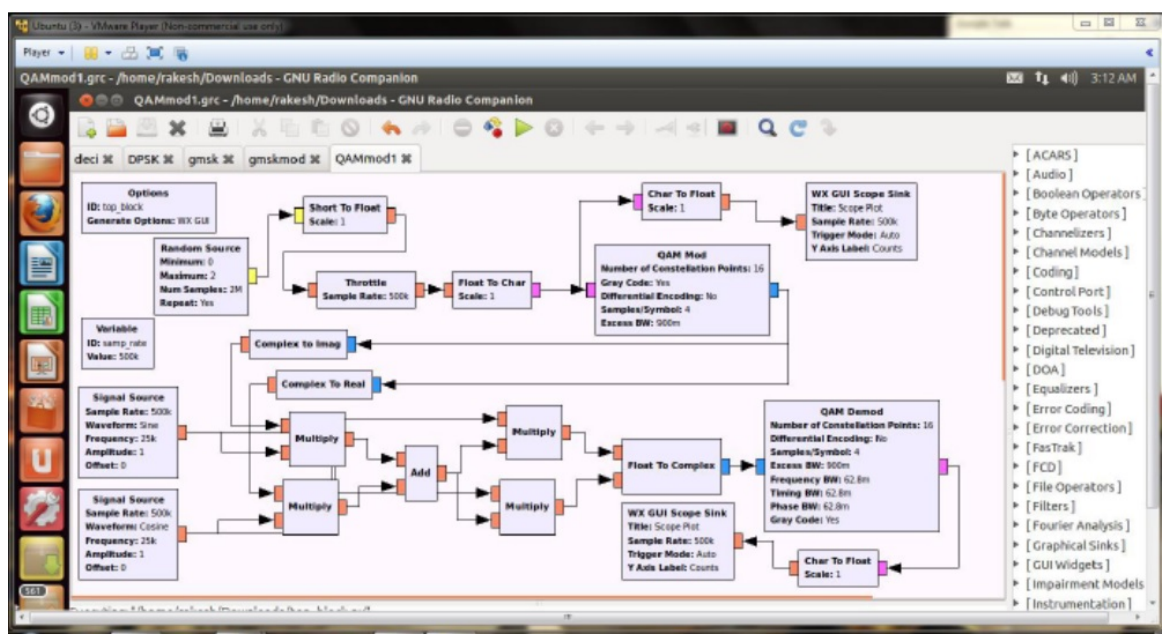

Fig. 1: Gráfico de simulación de un sistema con modulación QAM perteneciente al Lab1

En el Lab2 se introduce el uso de la plataforma USRP como transmisor en emisión FM (con una daughter-board Basic TX). El receptor está basado en RTL-SDR (p.e. NooElec SDR).

El Lab3 permite realizar un análisis experimental de las diferentes modulaciones monoportadora estudiadas en el Lab1, analizando su respuesta en función del BER y midiendo el SNR que se le aplica. La figura 3 muestra la maqueta que utilizamos para la demostración de funcionamiento del transmisión con modulación QPSK.

\footnotetext{
${ }^{1}$ Los estudiantes estudiarán los aspectos más relevantes de los estándares considerados e instalarán alguno de los proyectos correspondientes que se encuentran en la web http://www.cgran.org
} 


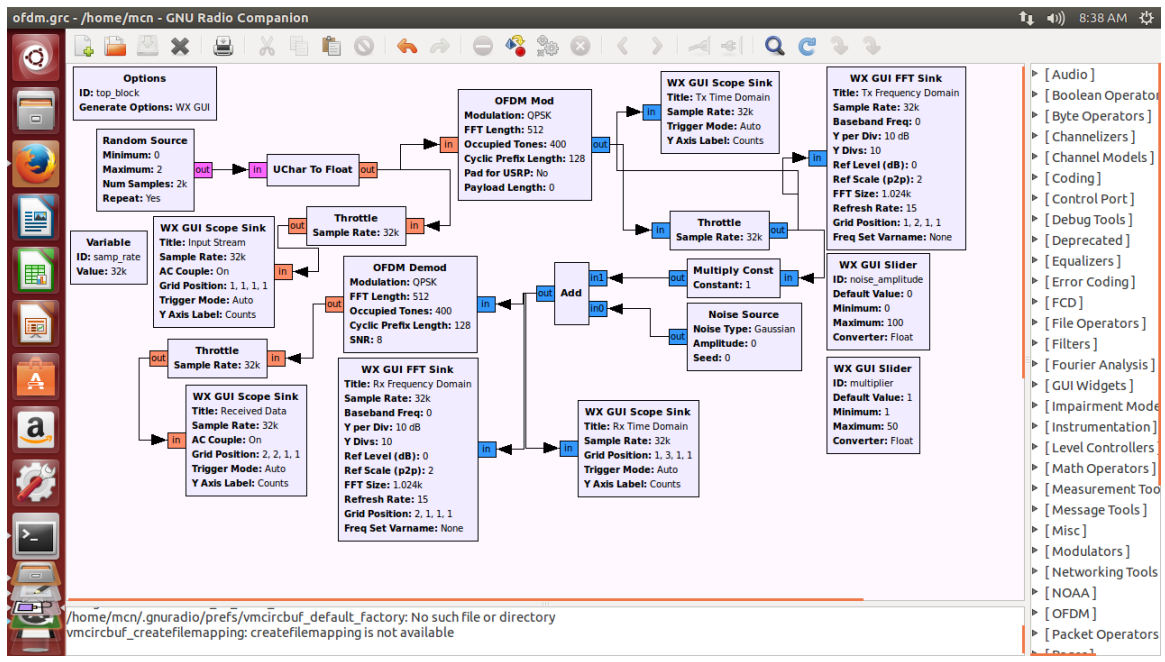

Fig. 2: Gráfico de simulación de un sistema con modulación OFDM perteneciente al Lab1

En el Lab4 se introduce una aplicación experimental de la modulación multiportadora que se analizó en la parte final del Lab1. Con ello se pretende estudiar qué características son aplicables a esta técnica y cómo actúan (p.e. precodificación cíclica, número de frecuencias portadoras, etc).

En el Lab5, como práctica que conjuga los diversos aspectos estudiados, se trata de forzar a los estudiantes a aplicar los conceptos analizados en el contexto de algunos estándares de comunicación seleccionados (p.e. DVB-T, 802.11, etc).

\section{Conclusiones}

En este artículo se está explorando la situación docente de las asignaturas de comunicaciones en el Grado de Ingeniería Telemática y en el Máster de Telecomunicaciones de la ETSE de la Universitat de València. Para su evaluación se ha aplicado un cuestionario que ha sido respondido por 50 estudiantes de diversos grupos de esta titulación. Como se preveía, la respuesta de los estudiantes evidencia una necesidad de carga experimental en estas asignaturas.

Para el aumento de la carga experimental, se ha propuesto la introducción de algunas sesiones basadas en sistemas SDR en los laboratorios de estas asignaturas. Para el desarrollo de éstas, se ha elegido la plataforma USRP por su versatilidad.

Este proyecto aún no ha acabado y actualmente se han diseñado las sesiones prácticas y se encuentra en fase de experimentación por el grupo de estudiantes control. Su apli- 

de la UVEG

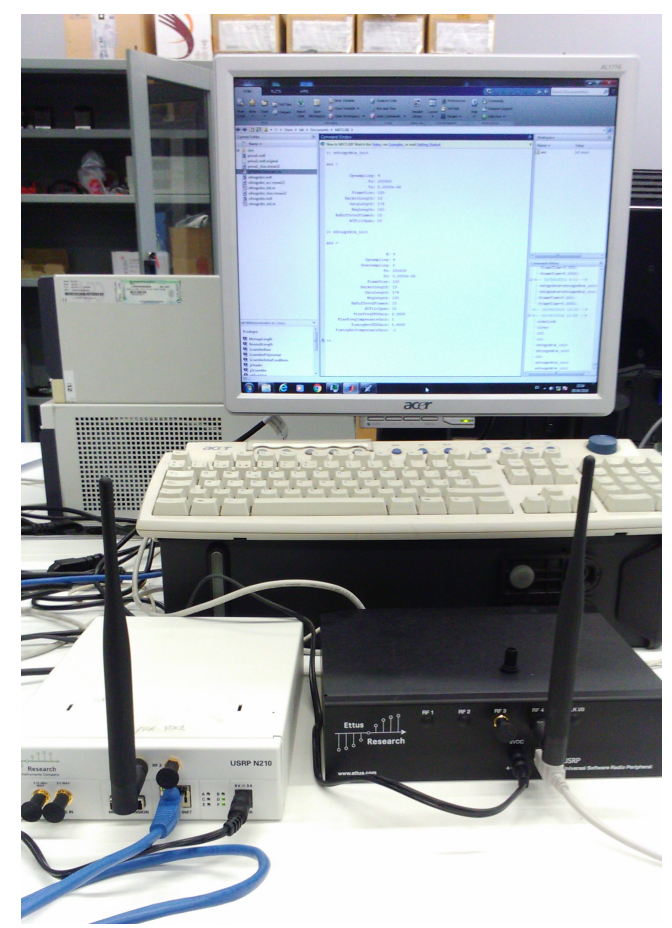

Fig. 3: Maqueta de una de las evaluaciones de transmisión con modulación QPSK perteneciente al Lab3

cación a los grupos implicados en las diferentes asignaturas será posterior, por tanto la respuesta del estudio final de los estudiantes del grado, que analiza la apreciación subjetiva después de la realización de las experiencias con USRPs, aún no ha sido recogida.

\section{Agradecimientos}

Los autores quieren agradecer al Servei de Formació Permanent i Innovació Educativa - Centre de Formació i Qualitat "Manuel Sanchis Guarner" de la Universitat de València por la ayuda concedida para el soporte parcial de este proyecto de innovación docente (ref: UV-SFPIE_RMD15-314373) y la Ministerio de Economía e Innovación por la ayuda con referencia TEC2013-47141-C4-4-R con la que se ha financiado parcialmente este trabajo. También quieren agradecer al grupo de estudiantes de grado que actúan como población control en este estudio de aplicación. 


\section{Referencias bibliográficas}

Aguaded-Gómez J I (2001). "Aprender y enseñar con las tecnologías de la comunicación". En: Agora digital 1.

Blázquez I (1994). "Nuevas tecnologías de la información y la comunicación". En: ed. por Blázquez F, Cabero J y Loscertales F. Sevilla: Alfar. Cap. Propósitos formativos de las nuevas tecnologías de la información y la comunicación en la formación de maestros, págs. 257-268.

Ettus Research (2015). Universal Software Radio Peripheral (USRP). (visitado en: 20/03/2016). URL: https://en.wikipedia.org/wiki/Universal_Software_ Radio_Peripheral.

Mao Sh y col. (2013). "Introducing Software Defined Radio into Undergraduate Wireless Engineering Curriculum through a Hands-on Approach". En: Proc. of the 120th ASEE Annual Conference 8 Exposition.

Mitola J (1992). "The software radio". En: Proc. IEEE National Telesystems Conference, pp.15-23, Washington, DC. (visitado en: 20/03/2016). URL: http:// courses.washington. edu/ee420/index.html.

Pinar-Domínguez I y Murillo-Fuentes J J (2011). Laboratorio de Comunicaciones Digitales Radio Definida por Software. (visitado en: 20/03/2016). TSC - Ed Universidad de Sevilla. URL: http://personal.us.es/murillo/docente/Libros/ LibroSDR.htm.

Wyglinski A M, Pu D y Cullen D J (2011). "Digital Communication Systems Education via Software-Defined Radio Experimentation". En: Proceedings of the 118th ASEE Annual Conference and Exposition Vancouver, BC, Canada. (visitado en: 20/03/2016). URL: http://courses.washington. edu/ee420/index.html. 